\title{
Clinical trials of Covid vaccines in adolescents: do the EMA and FDA have access to the same data?
}

\author{
Helene Banoun
}

Funding: The author(s) received no specific funding for this work.

Potential competing interests: The author(s) declared that no potential competing interests exist.

\section{Abstract}

Pfizer and Moderna vaccines just approved in Europe for children 12 years and older The EMA does not seem to have the same assessment as the FDA, which did not approve Moderna in the US.

The EMA and the FDA do not publish the same raw numbers regarding the effectiveness of the Pfizer vaccine.

Looking closely at the FDA data from the adolescent trial, it appears that in the crucial period between the first dose and 7 days after the second dose, the vaccine promotes Covid infections. Looking at the FDA report on the Pfizer adult trial conducted in 2020, the same phenomenon appears: worsening susceptibility to Covid in the post-vaccination period. The biological explanation of this phenomenon could be the ADE (antibody dependent enhancement) against which all official experts and critical scientists have been warning since March 2020 concerning Covid vaccines.

This clinical trial finding is consistent with the epidemiology of post-vaccine Covid outbreaks in the postmarketing phase.

The Moderna trial in adolescents shows clear efficacy and safety issues that likely prevented the FDA from validating it.

Regarding adverse events, the EMA and the FDA agree on the very high reactogenicity of these vaccines: this seems to increase as the target population ages, leading to fears of the worst if it is decided to vaccinate children from 6 months of age in the near future.

According to the manufacturer's predictions, the following effects can be expected with these vaccines: neurological, hematological and immune/autoimmune mediated vasculitis events; events associated with severe COVID-19 (i.e. ADE: aggravation of infection by vaccine antibodies, so this is implicitly recognized although never explained); severe thrombotic and embolic events.

The AEs observed and considered "expected" in 12-15 year olds are: pain at the injection site (in 86\% of adolescents after dose 2 ), fever ( $20 \%$ after dose 2 ), fatigue ( $60 \%$ of those vaccinated at dose 2 ), headache ( $55 \%$ of adolescents at dose $2=$ neurological effect), chills, arthralgia, myalgia, swelling at the injection site, nausea.

AEs are more common after the second dose. 
Serious AEs in the very small number of trial participants were neuralgia, paresthesia, lymphadenopathy, peritonitis/appendicitis, high fever, anaphylactic reaction, depression.

Since follow-up did not exceed 2 months for most participants, it is impossible to assess the long-term effects on these adolescents.

Given the benignity of Covid in adolescents, the benefit/risk ratio seems very unfavorable for the vaccine, knowing that it does not prevent transmission either and therefore cannot be considered altruistic.

FDA and EMA approval of Pfizer vaccine (Comirnaty) for adolescents aged 12-15 years EMA (but not FDA) approval of Moderna (Spikevax) vaccine for adolescents 12 years and older

\section{Introduction}

The mass vaccination campaign against Covid-19 began as early as December 2020 with mRNA vaccines that use a technology never before used on a large population and after only 6 months of unfinished clinical trials 1

Serious doubts remain about the efficacy and safety of these vaccines for adults. 2

On May 10, 2021, emergency approval was granted for the Pfizer BNT162b2 vaccine for persons older than 12 years 3 .

This approval was granted on the basis of a trial that observed the safety of the product only 1 month after the second dose on most participants (FDA report). The FDA recognizes that children and adolescents are unlikely to be infected with SARS-CoV-2 and experience mild Covid-19 most of the time. It is now established that the vaccine does not prevent transmission of the virus4.

Under these conditions, only a highly effective and safe vaccine should be licensed for adolescents: this does not appear to be the case.

\section{Pfizer trial in adolescents}

The FDA and EMA reports are based on the same randomized clinical trial conducted by Pfizer5 and published in the NEJM.6

The FDA and the EMA publish comparable results on the adverse effects of the Pfizer vaccine in adolescents, but the efficacy results are very discordant. 


\section{Adverse events}

According to the Risk Management Plan of April 20217, page 76, the expected risks of Pfizer vaccine are anaphylaxis, ADE - antibody dependent enhancement (VAED -vaccine-associated enhancement disease and VAERD - vaccine-associated enhancement respiratory disease), page 80 the list of expected AES according to those identified by experts on pharmacovigilance databases are: Immune/autoimmune-mediated neurological, hematological and vasculitis events; Events associated with severe COVID-19 (i.e. ADE: aggravation of infection by vaccine antibodies, so this is implicitly recognized although never explained)

Severe thrombotic and embolic events.

In the EMA report (Report published on May 28, 20218 many pages are rendered partially illegible by a black band applied over the details of the AEs (adverse events). This masking of data is not found on any other EMA document: would there be more to hide regarding trials on adolescents?

For example, on page 17, 13 adolescents aged 12 to 15 years (1131-1118) did not attend the first month visit after dose 2 and 7 were withdrawn between dose 1 and the end of the trial for a reason that is not visible, 66 young adults aged 16 to 25 years did not attend the 1 month visit after dose 2, idem we cannot see the number of adverse events responsible for these withdrawals. The same is true in the following pages up to page 50. It is noted that 2 adolescents left the trial for AEs but the details are hidden (also page 36).

For both the EMA and the FDA, the frequency of adverse events is higher in adolescents than in adults. These reactions are more frequent and more severe after the second dose.

They are pain at the injection site (86\% of adolescents after dose 2 ), fever (20\% after dose 2 in adolescents), especially after dose 2 , exhaustion ( $60 \%$ of those vaccinated at dose 2 ), headache (55\% of adolescents at dose 2 = neurological effect), chills, arthralgia, myalgia, swelling at the injection site, nausea.

Regarding severe AEs, one neuralgia and one paresthesia not related to the vaccine were noted by the investigators. Lymphadenopathy were found in significant numbers (7) in adolescents compared to young adults aged 16-25 years (only one): they are considered to be vaccine-related.

Between dose 1 and 1 month after dose 2, 4\% (or 7\%, it is not clear from Table 24) of adolescents had a serious AE (including 1 life-threatening), compared with 2\% of 16-25 year-olds (page 40).

AEs occurring after unmasking are not listed! So there were some!

Page 41: 72\% of 12-15 year olds had experienced an AE by the cut-off date (March 13, 2021); 33\% vaccine-related AEs, 7\% serious AEs, 1\% life-threatening. Again, data are masked in this Table 25. Two adolescents had a serious life-threatening AE: non-vaccine related peritonitis/appendicitis, high fever 
$\left(40.4^{\circ} \mathrm{C}\right)$; recall that in the adult group (16-55 years) 12 appendicitis were reported in the vaccine group versus 7 in the placebo group.

Two other adolescents in the placebo group who reached 16 years of age during the trial experienced severe life-threatening IE and were withdrawn from the trial. This was an anaphylactic reaction and depression; this depression was not related to the vaccine but to the hospitalization of this teenager, it is not specified why he was hospitalized following his first dose.

The EMA reminds us that "Covid-19 in adolescents is a mostly mild illness, and severe cases are also rare." These numerous serious IEs are therefore worrying and would tip the benefit/risk balance against the vaccine (as of June 24, 2021 in France, 8 children aged 0-14 years have died from Covid since March 1, 2020, without specifying comorbidity, Epidemiological Update COVID-19 No. 69 / Situation as of June 24, 2021 / p. 19, we read on the same bulletin of April 8, 2021, that the vast majority of the young people who died had comorbidities).

The EMA concludes that there is a lack of information in this trial for fragile patients with comorbidities (immunocompromised patients, patients with chronic obstructive pulmonary disease (COPD), diabetes, chronic neurological disease, cardiovascular disorders), patients with autoimmune or inflammatory disorders).

Also according to the EMA, "The study is not large enough to determine whether there is a rare adverse event with a higher frequency in adolescents compared to what has been observed in trials and real-life use in an older population."

"The long-term safety of the BNT162b2 mRNA vaccine is unknown at this time, but additional safety data are being collected in the ongoing study C4591001 up to 2 years after dose 2 of the BNT162b2 mRNA vaccine in all age groups. In addition, active surveillance studies are planned to monitor long-term safety in vaccinated individuals." Unfortunately if any of the placebo group is vaccinated when they reach age 16 , these data will be invalid. Surveillance is planned until the end of March 2024.

Pediatric population (the trial in this population is currently being launched9. Given the higher reactogenicity and higher antibody levels obtained in adolescents, the EMA recommends that the best dose suitable for children be sought in future trials (page 60) when vaccinating the pediatric population. Presumably, the dose injected in adolescents was too high and should have been adjusted before the trial began.

\section{Efficacy}

It is evaluated by immunogenicity (in vitro neutralizing antibody titers obtained after vaccination) and by protection against symptomatic Covid infection by comparing placebo and vaccinated groups. 


\section{Immunogenicity}

Participants were excluded from immunogenicity calculations without explanation (reason hidden by a black band): 71 excluded between 12 and 15 years and 94 between 16 and 25 years.

Antibody titers are higher in the adolescent group compared with the adult group, which is consistent with the higher reactogenicity of the vaccine in adolescents.

On page 24, it is stated that some participants in the placebo group achieve at least a 4-fold increase in neutralizing Ac titer 1 month after dose 2 of saline (29 in 12-15 year olds and 24 in 16-25 year olds, is this seroconversion due to Covid infection? It is not stated whether these individuals had symptoms and/or a positive PCR test). It is unfortunate that we do not have more quantitative data on these seroconversions due to natural infection with the virus to compare with vaccine seroconversions.

\section{Efficacy as measured by Covid cases averted in vaccinees}

A case of Covid is defined by a positive PCR test (without specifying Ct, cycle threshold) and 1 characteristic symptom (fever: from what temperature?, it is not specified, loss of taste, sore throat): this leaves the investigators free to define what is a case according to the placebo or vaccine group (Was the trial double-blind? It reads "observed-blinded" on the NIH website10 and "blinded" on the FDA report).. According to the EMA, page 25, there were 18 cases of Covid in the placebo group and 0 cases in the vaccinated group more than 7 days after dose 2 .

No efficacy is shown between dose 1 and 11 days after this dose (because the number of cases is small: 3 cases in the vaccinated group, 4 cases in the placebo group)

Page 27, between dose 1 and dose 2: 3 vaccinated cases and 12 placebo cases

Of which: After dose 1 and within 11 days: 3 vaccinated cases and 4 placebo cases

Between 11 days after dose 1 and before dose 2: 0 vaccinated cases and 8 placebo cases

After dose 2 and within 7 days: 0 vaccinated and 5 placebo

More than 7 days after dose 2 (and less than 4 months after dose 2): 0 vaccinated cases and 18 placebo cases

A total of 35 cases were observed in the placebo group (incidence 3.1\%) and 3 in the vaccinated group (incidence $0.26 \%$ ).

According to the data of Ourworldindata11, between October 15, 2020 and March 13, 2021 (dates of the clinical trial), the rate of Covid in the USA was between 158 and 758 covid cases per million inhabitants per period of 7 days: $0.016 \%$ to $0.076 \%$, therefore a maximum of $0.61 \%$ over 2 months, the duration of the intermediate period of the trial.

Page 38 of the Pfizer protocol on phase 2/3 trials dating from 202012 states that the attack rate of Covid19 is estimated at $1.3 \%$ per year in the placebo group Why such a difference between the attack rates observed in the general population and estimated in the 
trial (respectively $0.61 \%$ and $1.3 \%$ ) on the one hand and observed in the trial in the placebo group on the other hand $(3.1 \%)$ ?

Let's compare the EMA assessment with the one done by the FDA13.

For the FDA: in the period between dose 1 and 7 days after dose 2, 114 people were infected in the vaccine group and 132 in the placebo group (these are the people with obvious infection in this period) Why such a difference in numbers between EMA and FDA for the same trial and the same data? Would the FDA have other data available than the EMA?

\begin{tabular}{|c|c|c|c|}
\hline Disposition & $\begin{array}{r}\text { BNT162b2 } \\
\text { n(\%) }\end{array}$ & $\begin{array}{r}\text { Placebo } \\
\mathbf{n}(\%)\end{array}$ & $\begin{array}{l}\text { Total } \\
\mathbf{n}(\%)\end{array}$ \\
\hline Randomized $^{\mathrm{a}}$ & $1134(100.0)$ & $1130(100.0)$ & $2264(100.0)$ \\
\hline Dose 1 all-available efficacy population & $1131(99.7)$ & $1129(99.9)$ & $2260(99.8)$ \\
\hline $\begin{array}{l}\text { Participants without evidence of infection before } \\
\text { Dose } 1\end{array}$ & $1028(90.7)$ & $1023(90.5)$ & $2051(90.6)$ \\
\hline $\begin{array}{l}\text { Participants excluded from Dose } 1 \text { all-available efficacy } \\
\text { population }\end{array}$ & $3(0.3)$ & $1(0.1)$ & $4(0.2)$ \\
\hline \multicolumn{4}{|l|}{ Reason for exclusion ${ }^{b}$} \\
\hline Did not receive at least 1 vaccination & $3(0.3)$ & $1(0.1)$ & $4(0.2)$ \\
\hline Dose 2 all-available efficacy population & $1123(99.0)$ & $1117(98.8)$ & $2240(98.9)$ \\
\hline $\begin{array}{l}\text { Participants without evidence of infection prior to } 7 \\
\text { days after Dose } 2\end{array}$ & $1008(88.9)$ & $983(87.0)$ & $1991(87.9)$ \\
\hline $\begin{array}{l}\text { Participants excluded from Dose } 2 \text { all-available efficacy } \\
\text { population }\end{array}$ & $11(1.0)$ & $13(1.2)$ & $24(1.1)$ \\
\hline \multicolumn{4}{|l|}{ Reason for exclusion ${ }^{b}$} \\
\hline Did not receive 2 vaccinations & $10(0.9)$ & $13(1.2)$ & $23(1.0)$ \\
\hline Unblinded prior to 7 days after Dose 2 & $1(0.1)$ & 0 & $1(0.0)$ \\
\hline Evaluable efficacy population & $1119(98.7)$ & $1110(98.2)$ & $2229(98.5)$ \\
\hline $\begin{array}{l}\text { Subjects without evidence of infection prior to } 7 \text { days } \\
\text { after Dose } 2\end{array}$ & $1005(88.6)$ & $978(86.5)$ & $1983(87.6)$ \\
\hline $\begin{array}{l}\text { Participants excluded from evaluable efficacy ( } 7 \text { days) } \\
\text { population }\end{array}$ & $15(1.3)$ & $20(1.8)$ & $35(1.5)$ \\
\hline
\end{tabular}

Details of table 5, page 14: concerns the period between dose 1 and 7 days after dose 2

In the vaccinated group out of 1119 subjects (population on which the efficacy is assessable), only 1005 are retained (subjects without evidence of infection before 7 days after dose 2), therefore 1119-1005 = 114 subjects have evidence of infection before 7 days after dose 2, which represents an incidence of Covid in the vaccinated group of $114 / 1119=10.2 \%$;

In the placebo group, out of 1110 subjects (population on which the efficacy can be evaluated), 978 are retained (subjects without evidence of infection before 7 days after dose 2), which means that 132 were infected and the incidence rate of Covid in this placebo group is $132 / 1110=11.9 \%$.

The same remark can be made as for the EMA report: these incidence rates are extraordinarily high compared to those observed in the general population! 
Table 12, page 21, concerns the period after 7 days after the second dose 16 cases of Covid in the placebo group, thus 16/978 = incidence of $1.64 \%$. and zero cases in the vaccinated $0 / 1005=0 \%$ incidence

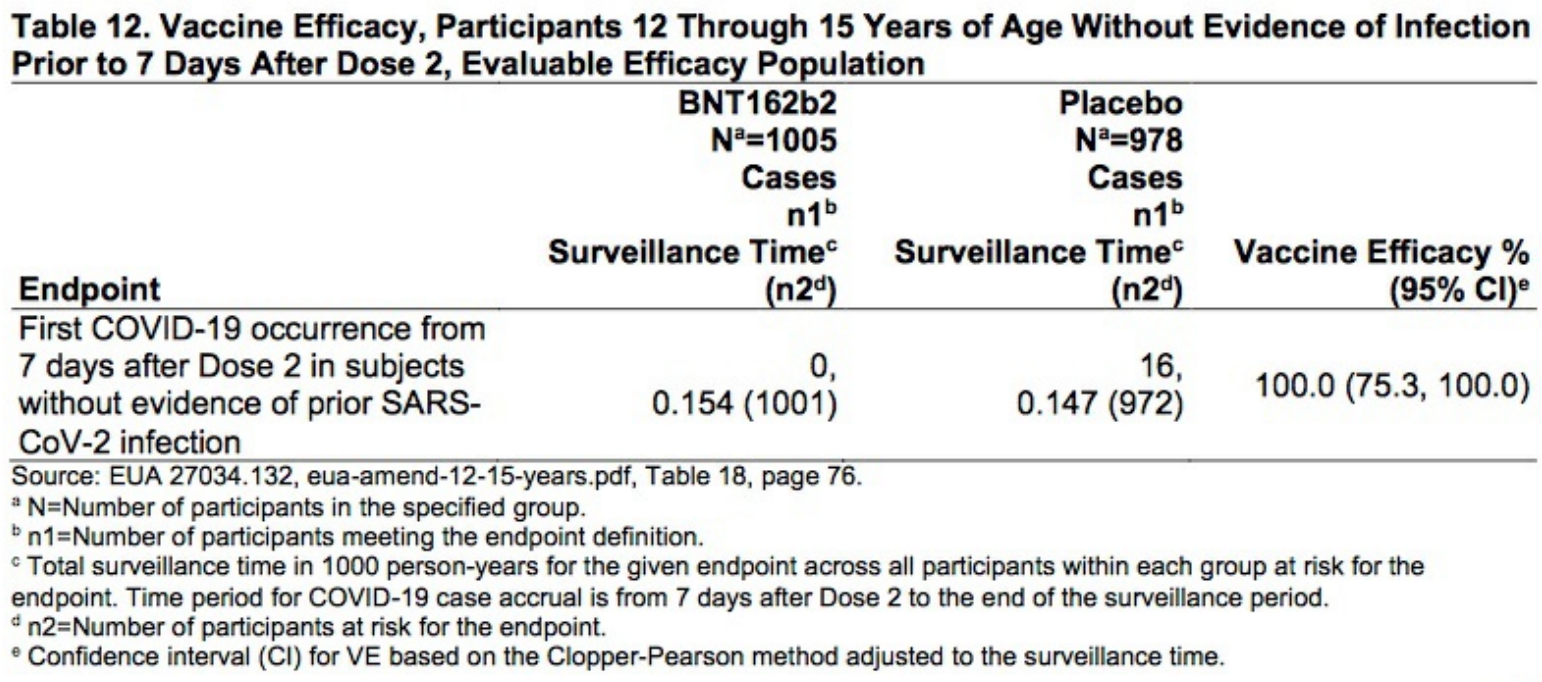

Let us compare the incidences of Covid in the two groups according to the two periods

In the vaccine group more than 7 days after dose 2 , the incidence is $0 \%$, so the protection of the vaccine against the absolute risk observed just before this period is $10.2 \%-0 \%=10.2 \%$.

In the placebo group, more than 7 days after the second dose of salt water, the protection is $11.9 \%$ -

$1.64 \%=10.26 \%$, so the placebo would protect slightly more than the vaccine?

How to explain the sharp drop in incidence in the placebo group 7 days after the 2 nd dose of salt water? How can we explain these extraordinarily high incidence rates in the period between dose 1 and 7 days after dose 2 compared to those observed in the general population?

The biological explanation could be ADE (antibody dependent enhancement): vaccine antibodies promote the entry of the virus into the cells instead of slowing it down and this mainly just after the first dose when they are at low levels and low affinity14. What could be the explanation for the high incidence also in the placebo group in this post-vaccination period? The FDA should comment on this.

Thus, according to the data reported by the FDA, it can be assumed that ADE is a risk associated with the COVID vaccine.

Neither the FDA nor the EMA explicitly mention it following the efficacy data.

However, the EMA highlights the significant risks identified (anaphylaxis) and the significant potential risks: vaccine-associated aggravated disease (VAED), including vaccine-associated aggravated respiratory disease (VAERD). And we know that these risks were mentioned in the Risk Management Plan following the previous adult trials.

But the EMA only recommends informing the public about the risk of anaphylaxis and about pregnancy and lactation. It does not recommend informing about the risks of ADE (VAED and VAERD). 
The EMA does not make any special recommendation for measures to minimize these serious risks which are risks on pregnancy and fertility, VAED and VAERD, long-term effects.

Note: I hadn't thought of doing these calculations for the 2020 adult trial, here they are, we see the same thing. The ADE was thus well highlighted although not explained on the 2020 trial; this is why it was notified in the risks to be expected on the RMP of the 2021 trial on adolescents.

\section{Addendum: ADE calculations for Pfizer adult trial 2020}

(page 18, table 2 of the FDA Pfizer Vaccines and Related Biological Products Advisory Committee Meeting December 10, 2020 - FDA Briefing Document Pfizer-BioNTech COVID-19 Vaccine15)

People infected between dose 1 and 14 days after dose 2: 20556 - $18678=1888$ in the vaccine group (attack rate: $9.2 \%$ )

Placebo group: 20536-18563 = 1973 (attack rate 9.6\%)

Same calculations for 7 days after dose 2

20566-18701 = 1865 (attack rate 9.1\%) Vaccinated group ;

$20536-18627=1909$ (attack rate $=9.3 \%$ ) placebo group

People infected from 7 days after dose 2:

Vaccinated: 8 (8/17400 participants: attack 0.046\%), absolute efficacy: $9.1-0.046=9.054 \%$

Placebo: $162(162 / 17511=0.93 \%$ attack rate), absolute efficacy of salt water: $9.3-0.93=8.37 \%$

Here, therefore, the placebo protects slightly less than the vaccine, which seems logical. However, we cannot explain this sudden drop in the attack rate between the two periods observed; nor can we explain the difference with the incidence of the disease in the general population (on November 14, 2020, 447 confirmed cases per million over a period of 7 days: $0.04 \%$, i.e., $0.32 \%$ over 2 months, the duration of the trial) or with the attack rate provided for in the protocol (1.3\%).

\section{Moderna vaccine trial in adolescents}

In June 2021, Moderna applied to the FDA for approval of its vaccine for adolescents.16

On August 11, 2021 the results of the clinical trial (Evaluation of mRNA-1273 SARS-CoV-2 Vaccine in Adolescents DOI: 10.1056/NEJMoa2109522) were published in the NEJM. On August 12, this vaccine is still reserved according to the FDA for those over 18 years of age, the FDA has not yet made a decision.17 On July 23, 2021, the EMA authorizes Moderna for children over 12 years.18.

Why has the FDA not yet approved Moderna for 12-17 year olds?

Presumably, the FDA was aware of additional unpublished data that did not allow them to approve this product. However, based on the published data, there are clear efficacy and safety concerns.

\section{Efficacy}

Reading the published data on this trial, it is clear that efficacy has not been proven. 
"COVID-19 was defined as symptomatic COVID-19 requiring a positive RT-PCR result and at least 2 systemic symptoms or 1 respiratory symptom. Cases are counted from 14 days after the second dose. There were zero cases of symptomatic COVID-19 in the Spikevax group and 4 cases of symptomatic COVID-19 in the placebo group."

Again, why not account for all cases from the 1st injection? Vaccination is carried out during the period of viral circulation, thus exposing the vaccinees to the risk of $A D E$, and this crucial period should be taken into account.

In the period between the first dose and 14 days after the second dose, 3 different categories of Covid infection are listed (as well as 14 days after dose 2 ).

In the first period 84 Covid cases are found in the placebo group (84/1240 $=6.8 \%$ incidence) and 54 in the vaccine group $(54 / 2486=2.2 \%)$

More than 14 days after dose 2, 50 cases were found in the placebo group (4\%) and 44 in the vaccinated group (1.8\%).

The supplemental material attached to the trial publication19 that the efficacy analysis was performed on 3,181 participants (2,139 vaccinated and 1,042 placebo), after exclusion of 347 vaccinated participants and 198 in the placebo group: why these exclusions? Nothing is specified, we cannot exclude postvaccination Covid, probably due to the ADE. This problem already existed for the first clinical trial published and reviewed by the FDA in December 202020 participants had been excluded from the study between the 2 doses.

\section{Safety}

The EMA warns of the risk of myocarditis and pericarditis especially in young men and after the second dose.

Reading the published information on this trial, it is clear that safety is not assured.

Lymphadenopathies, headaches, myalgias and arthralgias, high fevers, nausea and vomiting are very frequently encountered after the vaccine; anaphylaxis and hypersensitivity are noted at an "unknown" frequency! Facial paralysis and severe facial swelling have also been reported; all this in only 2486 vaccinated participants, which may predict many severe AEs in the marketing phase.

Pediatric population

The safety and efficacy of Spikevax in children and adolescents under 12 years of age have not yet been established. No data are available.

Toxicity

The new lipid SM-102 has been tested and the results suggest that the toxicity in humans should be low, carcinogenicity has not been tested. Clearly nothing is known about the toxicity of this new component.

\section{Conclusion}


The efficacy and safety of both Moderna and Pfizer vaccines are not clearly established in the clinical trials published to date. Given the benignity of Covid in adolescents, the benefit/risk ratio seems very unfavorable for the vaccine, knowing that it does not prevent transmission either and therefore cannot be considered altruistic.

\section{References}

1 (Vaccines and Related Biological Products Advisory Committee Meeting December 10, 2020 FDA Briefing Document Pfizer-BioNTech COVID-19 Vaccine https://www.fda.gov/media/144245/download)

2 (Doshi P. Covid-19 vaccines: In the rush for regulatory approval, do we need more data? BMJ 2021; 373:n1244 doi:10.1136/bmj.n1244)

3 ( Frenck RW, Klein NP, Kitchin N. Safety, Immunogenicity, and Efficacy of the BNT162b2 Covid-19 Vaccine in Adolescents. NEJM, online op 27/05/21. Doi: 10.1056/NEJMoa2107456)

4 (Outbreak of SARS-CoV-2 Infections, Including COVID-19 Vaccine Breakthrough Infections, Associated with Large Public Gatherings - Barnstable County, Massachusetts, July 2021

https://www.cdc.gov/mmwr/volumes/70/wr/mm7031e2.htm; Covid-19 Breakthrough Infections in Vaccinated Health Care Workers https://doi.org/10.1056/NEJMoa2109072).

5 (https://clinicaltrials.gov/ct2/history/NCT04649151)

6 (Frenck RW Jr, Klein NP, Kitchin N, Gurtman A, Absalon J, Lockhart S, Perez JL, Walter EB, Senders S, Bailey R, Swanson KA, Ma H, Xu X, Koury K, Kalina WV, Cooper D, Jennings T, Brandon DM, Thomas SJ, Türeci Ö, Tresnan DB, Mather S, Dormitzer PR, Şahin U, Jansen KU, Gruber WC; C4591001 Clinical Trial Group. Safety, Immunogenicity, and Efficacy of the BNT162b2 Covid-19 Vaccine in Adolescents. N Engl J Med. 2021 Jul 15;385(3):239-250. doi: 10.1056/NEJMoa2107456. Epub 2021 May 27. PMID: 34043894; PMCID: PMC8174030.).

7 (https://www.ema.europa.eu/en/documents/rmp-summary/comirnaty-epar-risk-management-plan_en.pdf) 8 https://www.ema.europa.eu/en/documents/variation-report/comirnaty-h-c-5735-ii-0030-epar-assessmentreport-variation_en.pdf)

9 https://www.covidvaccinestudy.com/pediatric-study)

10 https://clinicaltrials.gov/ct2/show/NCT04368728

11 (https://ourworldindata.org/covid-vaccinations), 
12 (https://cdn.pfizer.com/pfizercom/2020-11/C4591001_Clinical_Protocol Nov2020.pdf)

13 https://www.fda.gov/media/148542/download

14 (The potential danger of suboptimal antibody responses in COVID-19

https://www.nature.com/articles/s41577-020-0321-6, Informed consent disclosure to vaccine trial subjects of risk of COVID-19 vaccines worsening clinical disease,

https://www.ncbi.nlm.nih.gov/pmc/articles/PMC7645850/)

15 https://www.fda.gov/media/144245/download

16 (https://www.webmd.com/vaccines/covid-19-vaccine/news/20210614/moderna-asks-fda-for-adolescentcovid-vaccine-approval).

17 (https://www.fda.gov/emergency-preparedness-and-response/coronavirus-disease-2019-covid19/moderna-covid-19-vaccine)

18 old https://www.biopharma-reporter.com/Article/2021/07/23/Commission-backs-use-of-Modernavaccine-for-children-ages-12-and-over

https://www.ema.europa.eu/en/documents/product-information/spikevax-previously-covid-19-vaccinemoderna-epar-product-information_en.pdf (document published on July 31, 2021 by the EMA)

19 (Supplement to: Ali K, Berman G, Zhou H, et al. Evaluation of mRNA-1273 SARS-CoV-2 vaccine in adolescents. N Engl J Med. DOI: 10.1056/NEJMoa2109522 )

20 (https://www.fda.gov/media/144585/download FDA Review of Efficacy and Safety of Moderna COVID-19 Vaccine - Emergency Use Authorization Request): 